\title{
Molecular fluorescence lifetime fluctuations: on the possible role of conformational effects
}

\author{
R.A.L. Vallée ${ }^{\mathrm{a}, \mathrm{b}, *}$, G.J. Vancso ${ }^{\mathrm{b}}$, N.F. van Hulst ${ }^{\mathrm{a}}$, J.-P. Calbert ${ }^{\mathrm{c}}$, \\ J. Cornil ${ }^{c, d}$, J.L. Brédas ${ }^{c, d}$ \\ a Applied Optics Group, Research Institute, University of Twente, P.O. Box 217, 7500 AE Enschede, Netherlands \\ ${ }^{\mathrm{b}}$ Materials Science and Technology of Polymers MESA+ Research Institute, University of Twente, P.O. Box 217, \\ 7500 AE Enschede, Netherlands \\ ${ }^{\mathrm{c}}$ Laboratory for Chemistry of Novel Materials, University of Mons-Hainaut, Place du Parc 20, B-7000 Mons, Belgium \\ d Department of Chemistry, The University of Arizona, 1306 East University Blvd, Tucson, Arizona 85721-0041, USA
}

Received 21 November 2002; in final form 27 February 2003

\begin{abstract}
The radiative lifetime of single $1,1^{\prime}$-dioctadecyl-3,3,3',3'-tetramethylindodicarbocyanine molecules, embedded in a polymer thin film, has been characterized. At room temperature the chemically identical molecules exhibit strong fluctuations in their fluorescence lifetime. The possible conformational origin of these fluctuations has been addressed by semi-empirical quantum-chemical calculations. Specifically, the impact of conformational effects induced by thermal motion on the transition energy and the value of the transition dipole moment has been studied. The results indicate that the strong fluctuations cannot be explained by pure thermally activated conformational changes. Since quenching mechanisms are not responsible for the observed fluctuations, we suggest that molecular segmental dynamics of the surrounding polymer play a key role in determining the lifetime fluctuations.
\end{abstract}

(C) 2003 Elsevier Science B.V. All rights reserved.

\section{Introduction}

Single molecule spectroscopy (SMS) is a new powerful method, which allows to investigate the interactions between an individual fluorophore and its surrounding environment [1]. In the last

\footnotetext{
${ }^{*}$ Corresponding author. Present address: Laboratory of molecular dynamics and spectroscopy, Department of Chemistry, Katholieke Universiteit Leuven, Celestijnenlaan $200 \mathrm{~F}$, B-3001 Heverlee (Leuven), Belgium. Fax: +32-16-327990.

E-mail address: renaud.vallee@chem.kuleuven.ac.be(R.A.L. Vallée).
}

decade, much attention has been paid to the interesting observation that chemically identical single molecules embedded in the same matrix may show completely different behavior. In particular, distributions of lifetimes for terrylene molecules embedded in polyethylene at a temperature of $30 \mathrm{mK}$ have been observed with a relative standard deviation of about 20\% [2]. For nonpolar fluorophores, embedded in nonpolar polymer matrices, the distribution of radiative lifetimes has been suggested to result from the dispersion interaction, which is also responsible for inhomogeneous broadening and the solvent shift of the transition 
frequency [3]. This can be rationalized by the fact that the electronic energy states of a dye embedded in a host material are very sensitive to the local environment.

The transition frequency $\omega(t)$ of a specific transition of a single fluorophore is the sum of a static component $\omega_{0}$ and a dynamic component $\delta \omega(t)$. The variation in the static component originates from the fact that each molecule interacts with a different configuration of polymer segments, which shifts the value of its resonance frequency in a unique manner. The variation in the dynamic component results from the coupling with the fluctuating surrounding medium. Since glassy polymers always contain a significant amount of disorder, different fluorophores have different local environments and therefore will have different static and dynamic components (related to conformational changes of the dye induced by thermal motion and/or the dynamic of the surrounding polymer matrix) of their transition frequencies [3]. At room temperature, radiative lifetime distributions are wider than the cryogenic distributions, as other phenomena can take place inside the matrix when the temperature is increased. In particular,

(a)
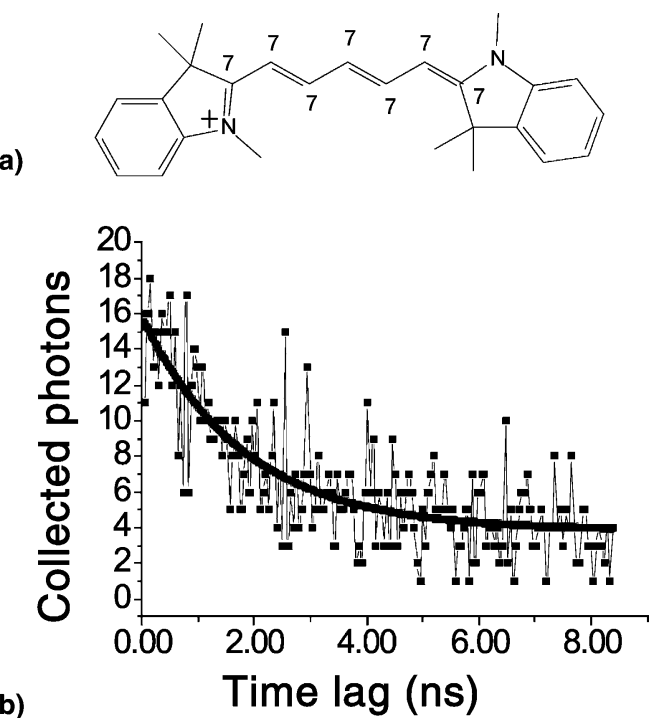

Fig. 1. (a) Chemical structure of the DiD fluorophore. (b) Fluorescence lifetime decay of a DiD fluorophore embedded in a $30 \mathrm{~nm}$ thick poly(styrene) film, at room temperature. The excitation power is about $3 \mathrm{~kW} / \mathrm{cm}^{2}$. the aperture of extra decay channels in the vicinity of the probe molecule is well-known to produce large fluctuations in lifetime [4].

In this Letter we report on fluorescence lifetime measurements performed at room temperature on 1,1'-dioctadecyl-3,3,3',3'-tetramethylindodicarbocyanine perchlorate (DiD) fluorophores (Fig. 1a) embedded in a polystyrene (PS) matrix. We observe that the lifetime of an individual fluorophore can vary up to $100 \%$ with respect to its mean value. The results of our quantum-chemical calculations indicate that conformational changes are not responsible for the extremely large lifetime fluctuations observed. Furthermore, our data clearly show that the fluctuations do not arise from quenching mechanisms.

\section{Experimental}

Experimental details can be found elsewhere [5]. In brief, dye-doped polymer thin films $(30 \mathrm{~nm})$ were prepared by spin-coating of a solution of $\operatorname{DiD}\left(5 \times 10^{-10} \mathrm{M}\right)$ and PS (number average molar mass of $89300 \mathrm{~g} / \mathrm{mol}$, polydispersity index of 1.06 ) in toluene onto a glass substrate. Annealing was performed in order to relax the stresses induced by the deposition procedure [5]. The films were investigated using a confocal scanning fluorescence microscope illuminated by femtosecond laser pulses at a repetition rate of $80 \mathrm{MHz}$. Time correlated single photon counting allows for the acquisition of fluorescence lifetime transients.

\section{Results and discussion}

After photoexcitation of a probe molecule in its first excited state, the system can relax by either radiative or nonradiative decay channels in a few nanoseconds. Integrating the process during a $200 \mathrm{~ms}$ time bin allows us to build an histogram of the time-lags between the excitation and fluorescence processes. Fig. $1 \mathrm{~b}$ shows such an histogram, best-fitted by a single-exponential decay with a fluorescence lifetime $\tau_{\mathrm{f}}=1.814 \mathrm{~ns}$. Repeating the experiment, we can monitor the temporal and local fluctuations of the local environment around the 
probe. Fig. 2 shows transients of fluorescence lifetime and fluorescence intensity for two individual DiD molecules embedded in a $30 \mathrm{~nm}$ thick film of PS. These two molecules are representative of hundreds of others, observed in the same conditions. In both cases, the intensity weakly fluctuates, within a $10-20 \%$ range, around the mean value; note the latter is larger for molecule a than for molecule $b$ since the fixed dipoles of these two molecules are not oriented in the same way with respect to the incident circularly polarized beam [5]. However, while the fluorescence lifetime of the first molecule varies very little and rather symmetrically around the mean value, the second molecule exhibits a lifetime that makes frequent excursions towards higher values and reaches
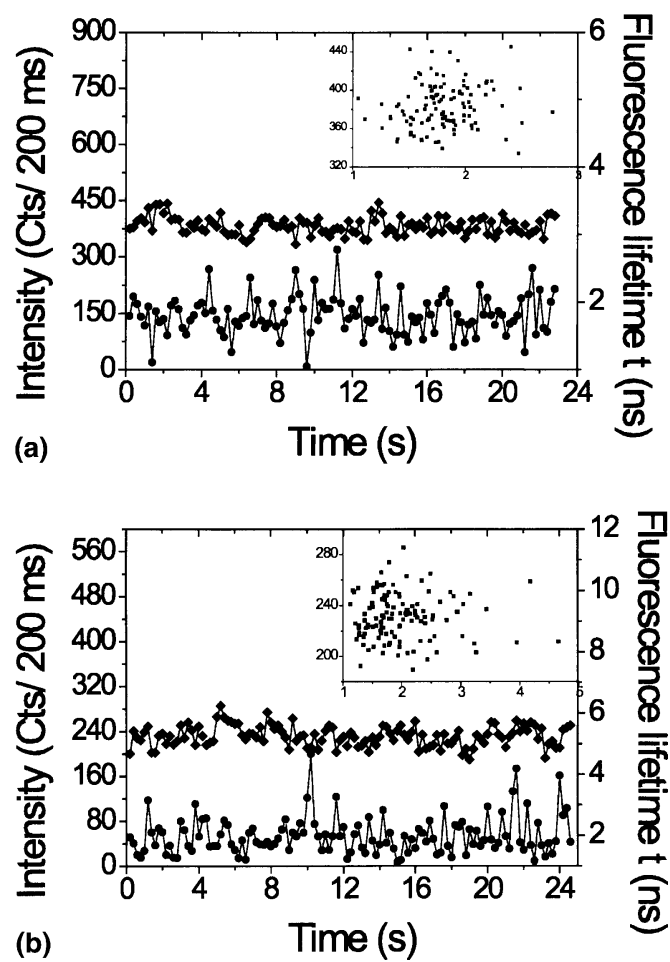

Fig. 2. Intensity (diamonds) and fluorescence lifetime (circles) trajectories of two different DiD molecules embedded in a $30 \mathrm{~nm}$ thick film of polystyrene, at room temperature and with an excitation power of about $3 \mathrm{~kW} / \mathrm{cm}^{2}$. The second molecule (b) shows peculiar excursions towards higher lifetimes. The insets show correlation plots between intensity and lifetime. They show no correlation, indicating no aperture of extra decay channels occurring during the measurement.
$100 \%$ deviation above the mean value. What can be the origin of such lifetime fluctuations? We mentioned previously that quenching mechanisms can play an important role in a polymer matrix at room temperature. However, the insets of Fig. 2 show the absence of correlation between intensity and fluorescence lifetime, for each molecule. Combining this observation with the fact that fluctuations lifetime point towards higher values and that DiD has a high quantum efficiency (close to unity) allows us to exclude the activation of an efficient nonradiative decay channel. A second entry to the problem is to examine the parameters of the decay rate formula [6]:

$\tau_{10}^{-1}=\sqrt{\varepsilon} \frac{\omega_{10}^{3} d_{10}^{2}}{3 \pi \varepsilon_{0} \bar{h} c^{3}}$,

where $\tau_{10}, \omega_{10}, d_{10}, \varepsilon_{0}$ and $\varepsilon$ designate the radiative lifetime, transition angular frequency, and transition dipole moment of the excited state of the fluorophore for the $S_{1}-S_{0}$ transition, and the dielectric constants of the vacuum and of the considered medium $(\varepsilon=2.5$ for polystyrene), respectively. This equation shows that fluctuations in transition frequency and transition dipole moment can be responsible for the observed changes in lifetime. In this context, we have performed quantum-chemical calculations to assess the impact of conformational fluctuations on the fluorescence lifetime.

We have first optimized the geometry of the molecule in the ground state using the semiempirical Hartree-Fock Austin Model 1 (AM1) method [7] and in the lowest excited state by coupling the AM1 Hamiltonian to a full configuration interaction (CI) scheme within a limited active space, as implemented in the Ampac package [8]. The equilibrium geometry of the isolated molecule is close to planarity and displays very similar bond lengths and bond angles in the two states; the latter behavior is related to the fact that both states are described by a superposition with equal weight of the two resonance forms of the cyanine backbone [9]. This also implies that transition dipole moments associated with absorption and emission processes have a similar magnitude.

We have then computed the optical absorption spectra of the molecule by means of the semi- 
empirical Hartree-Fock intermediate neglect of differential overlap (INDO) method, as parameterized by Zerner et al. [10], combined to a single configuration interaction (SCI) technique; the CI active space is built here by promoting one electron from one of the highest twenty occupied to one of the lowest twenty unoccupied levels. The first excited state giving rise to the lowest absorption band, is calculated at $2.27 \mathrm{eV}$, in good agreement with the experimental value around $1.90 \mathrm{eV}$ [5]; the slight discrepancy between the two values most likely originates from the neglect of medium effects in the theoretical approach. The first excited state is mostly described by an oneelectron transition between the highest occupied molecular orbital (HOMO) and lowest unoccupied molecular orbital (LUMO) levels, whose bondingantibonding pattern is illustrated in Fig. 3. These two levels are delocalized over the whole molecular backbone with dominant weights found over the polyenic segment. Interestingly, the electronic density is mostly localized on the [odd-numbered] even-numbered carbon atoms of the polyenic chain in the [LUMO] HOMO level. The transition dipole moment $\mu_{01}$, that can be written here as:

$\mu_{01}=\int \operatorname{HOMO}(r) r \operatorname{LUMO}(r) \mathrm{d} r$

is estimated to be $14.9 \mathrm{D}$ at the INDO/SCI level in the planar conformation. In view of the orbital shapes, it has vanishingly small contributions arising from the polyenic segment. This is illustrated in Fig. 4, which displays the atomic transition densities $q_{i}$ associated with the lowest excited

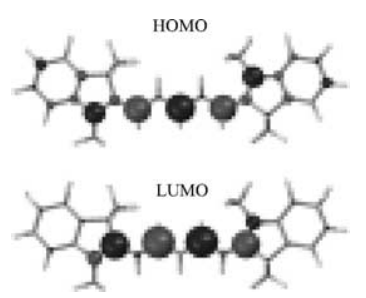

Fig. 3. Illustration of the linear combination of atomic orbitals (LCAO) pattern of the HOMO and LUMO levels of the DiD molecule, as calculated at the INDO level. The size and color of the balls reflect the amplitude and sign of the coefficients, respectively. The plots have been generated with the ZOA program [12].

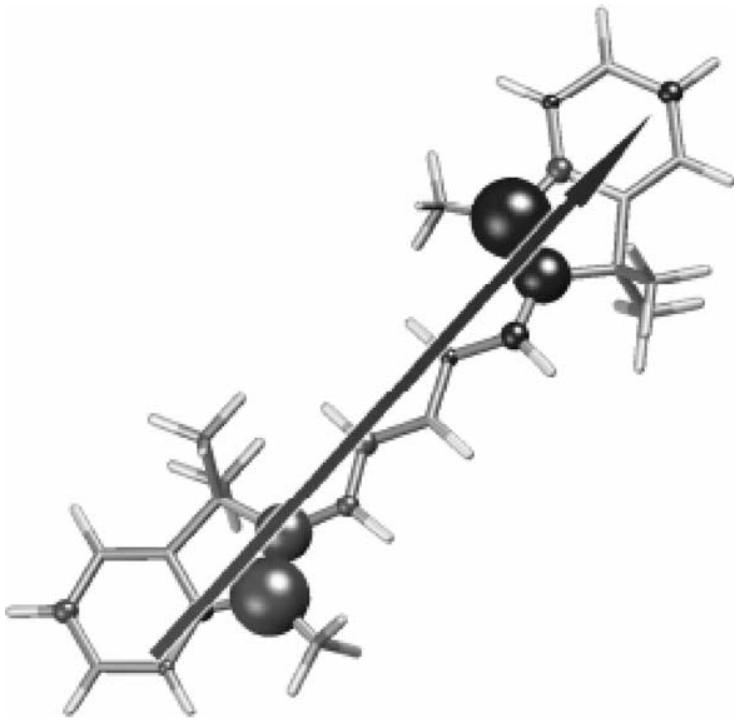

Fig. 4. Illustration of the distribution of the atomic transition densities associated to the transition between the ground state and the lowest excited state of the molecule, as calculated at the INDO/SCI level. The arrow describes here the orientation of the total transition dipole moment. The plot has been generated by the ZOA program [12].

states. Note that the atomic transition densities are calculated at the INDO/SCI level in a such a way that summing their values $q_{i}$ multiplied by the spatial coordinates $r_{i}$ of the corresponding atoms yields the total transition dipole moment $\mu$ [11]. Here, $\mu$ is found to be oriented along the main axis.

In order to assess the impact of possible conformational effects on the transition energy and transition dipole moments, we have first calculated at the AM1 level the potential energy curves associated to a rotation around the bond connecting the carbon atoms 6-7 and 4-5, respectively (see labeling in Fig. 1). These are displayed in Fig. 5 together with the corresponding evolution of the radiative lifetime obtained from Eq. (1) using the INDO/SCI-calculated transition dipole moments and transition energies obtained for each torsion angle. In both cases, the calculated curves show that the molecule can be twisted by up to $10^{\circ}-20^{\circ}$ with a few kTs, when taking a planar conformation as the starting geometry. These fluctuations will not significantly impact the radiative lifetime, 

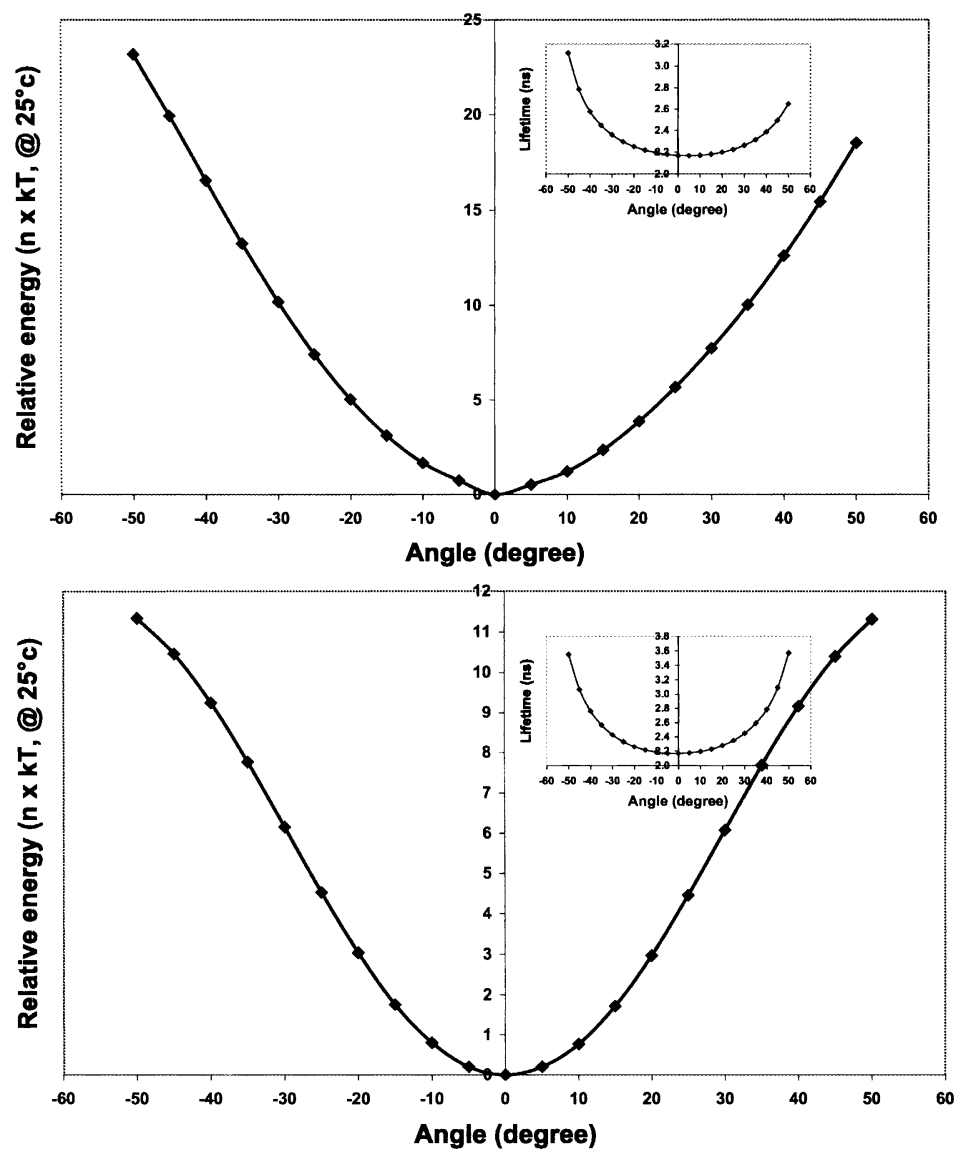

Fig. 5. AM1-calculated torsion potential profile associated to rotations around the 6-7 (top) and 4-5 (bottom) carbon atoms of the molecule. The insets illustrate the corresponding evolution of the radiative lifetime.

as evidenced by its calculated evolution as function of the rotational angle (see Fig. 5). In both cases, the values change by less than $10 \%$ between $0^{\circ}$ and $30^{\circ}$, which is in good agreement with the reported schematic diagram of potential energy surfaces involved in radiative deactivation of a single cyanine dye molecule [13]. Moreover, the flatness of these curves does not allow us to reproduce the large fluctuations observed experimentally, even when considering that the molecule is initially in a slightly twisted conformation, as a result of local, steric interactions with the surrounding polymer chains; doubling the radiative lifetime would actually requires a change in the torsion angle by more than $50^{\circ}$. Thus, the results of our calculations rule out that thermally activated conforma- tional changes are the main origin for the strong evolution in the radiative lifetime observed experimentally.

\section{Conclusions}

In summary, we have measured, by scanning confocal fluorescence microscopy, the fluorescence lifetime of DiD molecules embedded in a polystyrene matrix. The experimental data display lifetime fluctuations reaching $100 \%$; a priori this effect could be attributed to conformational changes of the molecule induced by thermal heating or to the dynamics of the surrounding medium. The results of quantum-chemical calculations, 
however, indicate that, upon rotation of the terminal fused rings by $20^{\circ}$ (i.e., when spanning new conformations accessible by thermal activation), the changes occurring in the transition energy and transition dipole moment associated to the emitting state are very small; hence, conformational fluctuations only lead to weak fluctuations in radiative lifetime. Thus, the strong disparity in fluorescence lifetimes observed experimentally cannot be mainly attributed to conformational dynamics of the probe molecule. This behavior cannot be attributed either to quenching effects in the matrix. Thus, the fluctuations in the observed lifetimes have to come from density fluctuations of the surrounding polymer.

\section{Acknowledgements}

R.A.L. Vallée is grateful to the Council for Chemical Sciences of the Netherlands Organization for Scientific Research (NWO-CW) for supporting this research. The work in Arizona is supported by the National Science Foundation (CHE-0078819), the Petroleum Research Fund, and the IBM Shared University Research Program. The work in Mons is supported by the Belgian Federal Government 'InterUniversity Attraction Pole in Supramolecular Chemistry and Catalysis (PAI 4/11)', and the Belgian National Fund for Scientific Research (FNRS-FRFC). J.C. is an FNRS research associate; J.P.C. acknowledges a grant from the Fonds pour la Formation à la Recherche dans l'Industrie et dans l'Agriculture (FRIA).

\section{References}

[1] T. Basché, W.E. Moerner, M. Orrit, U.P. Wild, Single Molecule Optical Detection, Imaging and Spectroscopy, Wiley-VCH, Weinheim, 1997.

[2] E.A. Donley, T. Plakhotnik, J. Chem. Phys. 114 (2001) 9993.

[3] E. Geva, J.L. Skinner, J. Phys. Chem. B 101 (1997) 8920.

[4] X.S. Xie, R. Dunn, Science 265 (1994) 361.

[5] R. Vallée, N. Tomczak, H. Gersen, E.M.H.P. van Dijk, M.F. Garca-Parajó, G.J. Vancso, N.F. van Hulst, Chem. Phys. Lett. 348 (2001) 161.

[6] R.J. Glauber, M. Lewenstein, Phys. Rev. A 43 (1991) 467.

[7] M.J.S. Dewar, E.G. Zoebisch, E.F. Healy, J.J.P. Stewart, J. Am. Chem. Soc. 107 (1985) 3902.

[8] AMPAC, Semichem, 7204 Mullen, Shawnee, KS 66216; the active space involves here the highest four occupied and lowest four unoccupied molecular orbitals.

[9] F. Meyers, S.R. Marder, B.M. Pierce, J.L. Brédas, J. Am. Chem. Soc. 116 (1994) 10703.

[10] M.C. Zerner, G.H. Loew, R. Kichner, U.T. MuellerWesterhoff, J. Am. Chem. Soc. 122 (2000) 3015.

[11] J. Cornil, D. Beljonne, J.P. Calbert, J.L. Brédas, Adv. Mater. 13 (2001) 1053.

[12] Zoa V2.0, J.P. Calbert, Laboratory for Chemistry of Novel Materials, University of Mons-Hainaut, Belgium.

[13] F. Kohn, J. Hofkens, R. Gronheid, M. Van der Auweraer, F.C. De Schryver, J. Phys. Chem. A 106 (2002) 4808. 